\title{
Análise da integração da recuperação da informação, information search behaviour e interação humano-computador para avaliação de sistemas de recuperação da informação'
}

\author{
The analysis of integration of information retrieval, information \\ search behaviour and human-computer interaction for the \\ assessment of information retrieval systems
}

Gracielle Mendonça Rodrigues GOMES²

Beatriz Valadares CENDÓN²

\section{Resumo}

Mesmo diante de várias mudanças para aperfeiçoar os sistemas de recuperação de informação, o último desafio da pesquisa na área ainda é proporcionar sistemas que sejam capazes de recuperar as informações relevantes disponíveis para melhor satisfazer a necessidade do usuário. Recentemente, no desenvolvimento de sistemas de recuperação da informação têm sido privilegiadas abordagens que considerem os aspectos humanos em recuperação da informação. Este artigo apresenta as áreas de Recuperação da Informação, Information Search Behaviour e Interação Humano-Computador, e discute a importância da sinergia entre elas para a avaliação e o desenvolvimento de sistemas altamente interativos. Além disso, evidencia que a interseção entre estas áreas formou uma especialidade de pesquisa denominada de Recuperação da Informação Interativa que tem como objetivo capacitar as pessoas a entender e a solucionar suas necessidades de informação através das interações com os sistemas.

Palavras-chave: Avaliação de sistemas de recuperação da informação. Comportamento do usuário. Interação homem-computador. Necessidades informacionais. Recuperação da informação.

\begin{abstract}
Even with several changes to improve information retrieval systems, the ultimate challenge of the research in the area is to provide systems that are able to retrieve relevant information available to best meet the users' needs. The developments in information retrieval systems have prioritized approaches that consider the human aspects in information retrieval. The aim of the article is to present the areas of research in Information Retrieval, Information Search Behaviour and Human-Computer Interaction and to discuss the importance of the synergy among them the assessment and development of highly interactive systems. In addition, shows that the intersection of these areas resulted in a research specialty called Interactive Information Retrieval that aims to enable people to understand and to solve their information needs through interactions with the systems.
\end{abstract}

Keywords: Information retrieval systems evaluation. User behaviour. Human-computer interaction. Information needs. Information retrieval.

\footnotetext{
1 Artigo elaborado a partir da dissertação de mestrado de G.M.R. GOMES, intitulada "Um estudo sobre a interação dos usuários com a interface e o sistema de busca do Portal de Periódicos da Capes". Universidade Federal de Minas Gerais, 2014

2 Universidade Federal de Minas Gerais, Escola de Ciência da Informação, Departamento de Organização e Tratamento da Informação. Av. Pres. Antônio Carlos, 6627, 31270-901, Belo Horizonte, MG, Brasil. Correspondência para/Correspondence to: G.M.R. GOMES. E-mail: <graciellemendonca@yahoo.com.br>. Recebido em 15/4/2014, reapresentado em 19/8/2014 e aceito para publicação em 8/10/2014.
} 


\section{Introdução}

Muitos usuários querem utilizar as vantagens da rapidez e da capacidade de recuperação dos Sistemas de Recuperação da Informação (SRI) automatizados. No entanto, existem problemas na concepção destes sistemas, pois os usuários destes sistemas ainda têm muitas dificuldades para usá-los no seu processo de busca de informação.

Particularmente com a disponibilidade de muitos recursos através da Web, torna-se importante compreender as experiências dos usuários na busca de informação em SRI no ambiente digital, e que naturalmente, implica em avaliar a interface, portanto a comunidade de pesquisa de Recuperação de Informação tem realizado o esforço de avaliar as interfaces de busca de informação para compreender o comportamento dos usuários e tornar mais fácil a elaboração de estratégias de pesquisas permitindo tanto aos usuários noviços quanto aos experientes realizar uma pesquisa mais rápida e eficiente.

A realização de estudos sobre como as pessoas se comportam e buscam informação através de SRI estão se tornando cada vez mais relevantes para melhorar a compreensão deste processo, além da perspectivas de trabalho em conjunto com outras disciplinas como, por exemplo, a Interação Humano-Computador.

O objetivo deste trabalho é apresentar revisão de literatura sobre alguns desafios da área de Recuperação da Informação (RI), a pertinência dos estudos de Information Search Behaviour, a constituição, conceitos e critérios de qualidade de uso da área de Interação Humano-Computador (IHC), e a importância da sinergia entre estas áreas para a avaliação e o desenvolvimento de sistemas altamente interativos. Além disso, busca-se evidenciar que a interseção entre estas áreas formou uma especialidade de pesquisa denominada de Recuperação da Informação Interativa (RII) que tem como objetivo capacitar as pessoas a entender e a solucionar suas necessidades de informação através das interações com os sistemas de recuperação da informação.

Para desenvolver esta investigação foi realizado o levantamento bibliográfico através de bases de dados da área de Ciência da Informação e áreas interdisciplinares disponíveis através do Portal de Periódicos Capes: Library and Information Science Abstracts; Library
Information Science \& Technology Abstracts; Emerald Fulltext; Scopus e Web of Science, além do Google Acadêmico.

\section{Desafios da recuperação da informação}

A eficiência de um SRI é avaliada pela capacidade de apresentar informações que atendam às necessidades dos usuários. Para isso, seus organizadores constroem um sistema que deve ser capaz de entender o que o usuário - a maioria das vezes inexperiente - quer. Por outro lado, a eficiência do usuário depende de sua capacidade de oferecer ao sistema elementos suficientes para que sejam selecionados, a partir da totalidade das informações armazenadas, um conjunto de itens que constituam a resposta que procura, como considera Branski (2004).

Souza (2006) afirma que nestes sistemas, há usualmente uma interface através da qual o usuário traduz sua necessidade de informações em forma de questões ou palavras-chave, ou mesmo examina os documentos na busca de informações pertinentes. Os dois modos de buscar informações são classificados como: modelos de recuperação, no qual o usuário propõe uma questão ou necessidade de informação ao sistema, e modelos de navegação, em que o usuário navega através dos documentos que não foram necessariamente indexados previamente, buscando informações de interesse.

No processo de recuperação da informação por meio de um sistema de busca, a experiência do usuário conta muito na construção da estratégia de busca ideal. Nesse sentido, Torres Pombert (2003) alerta que para se obter resultados significativos é necessário um misto de experiência, técnicas, habilidades, criatividade e boa sorte, tudo isso combinado com a capacidade de definir uma direção de forma metódica e clara, ou seja, navegar com uma finalidade.

O usuário deve avaliar o que conhece a respeito do tópico e o que pretende saber e, a partir daí, executar os seguintes passos: formular a questão da pesquisa e sua abrangência; identificar os conceitos importantes dentro da questão; definir a linguagem de busca que identifique estes conceitos; considerar sinônimos ou variações da linguagem; preparar a lógica da busca; ser capaz de elaborar a linguagem de busca e a estratégia adequada. 
Apesar do contínuo esforço dos desenvolvedores dos SRI para a criação de sistemas amigáveis, o processo de busca constitui, ainda, uma questão complexa. Até o momento o último desafio da pesquisa em Rl é proporcionar melhores sistemas que sejam capazes de recuperar as informações relevantes para melhor satisfazer a necessidade do usuário e nesta jornada os pesquisadores buscam desenvolver e utilizaram várias técnicas (Mamoon et al., 2013).

\section{Information search behaviour}

O comportamento de busca em sistemas de recuperação da informação, também denominado information search behaviour, é o comportamento empregado pelo usuário na interação com o sistema, no nível da interação humano-computador e no nível intelectual que também envolve atos mentais, como o julgamento de dados ou informações recuperadas. $\mathrm{O}$ information search behaviour é o subconjunto do comportamento de busca proposital de informação, o nível referido também como information-seeking behaviour, realizado pelo indivíduo para descobrir e ter acesso às fontes de informação. Este comportamento de busca de informação é um subconjunto do que pode ser definido como o campo mais geral de pesquisa, o comportamento informacional ou information behaviour, como expõe o modelo de Wilson (1999).

De acordo com Xie (2010), investigações recentes revelam a limitação da pesquisa no campo de RI tradicional no qual pressupõe que a busca de informações é estática e que as pessoas formulam um único tipo de consulta durante o processo de busca. No entanto, estas pesquisas têm mostrado que o processo de busca é dinâmico no qual as pessoas aplicam vários tipos de estratégias de busca.

Ainda existe a necessidade de uma orientação maior sobre como realizar as avaliações de SRI e de medidas válidas que reflitam uma variedade de situações de busca, como explicitam Kelly e Sugimoto (2013), à medida que mais pesquisadores começam a se concentrar no desenvolvimento de sistemas que suportam a busca e a recuperação de informação interativa. Todavia, o grau em que as teorias, objetivos e métodos em recuperação de informação interativa têm sido estabelecidos mostra que ainda foram pouco estudados.

Conforme Xie (2010, p.4), "a busca de informação em SRI's é afetada por diferentes tipos de fatores, sendo que quatro tipos são determinantes na escolha e aplicação de diferentes estratégias de busca":

1) A tarefa e o objetivo do usuário: a medida que aumenta o nível de complexidade da tarefa, mais canais e recursos de informação são necessários, e diferentes tipos de tarefas levam a aplicação de diferentes estratégias de busca.

2) As estruturas de conhecimento do usuário: o domínio do conteúdo ajuda os usuários a desenvolverem uma compreensão aprofundada das tarefas de busca, o conhecimento sobre a recuperação da informação e o sistema de busca auxilia no desenvolvimento de estratégias e para melhorar o desempenho da busca.

3) O contexto social e organizacional: o ambiente de trabalho influencia na forma como os usuários determinam suas estratégias e o processo de busca. Além disso, as dimensões culturais também intervêm em como os usuários interagem com os SRI.

4) O design do sistema de recuperação da informação: interfaces, ferramentas computacionais e os objetos informacionais são os principais componentes dos SRI's que orientam ou impedem usuários de aplicar diferentes estratégias de busca.

Para representar os padrões de busca de informação, vários modelos de busca têm sido desenvolvidos para ilustrar tanto o processo dinâmico da busca como os principais elementos que afetam o processo. Segundo Joseph et al. (2013), a identificação e o desenvolvimento de modelos de busca de informação têm oferecido orientações importantes sobre a forma como os usuários interagem com seus ambientes de informação, portanto os profissionais da informação podem utilizar a avaliação do comportamento de busca para melhorar o atendimento das necessidades dos usuários e rever o projeto de sistemas de informação.

\section{Interação humano-computador}

O uso e o acesso das tecnologias computacionais não têm acontecido com o sucesso que se esperava. 
Vários fatores como falta de infraestrutura, problemas sociais, econômicos, políticos, bem como a inexperiência, falta de habilidade e incapacidade do usuário, falta de treinamento e dificuldades criadas até mesmo pelos próprios desenvolvedores destas tecnologias têm criado barreiras para o manuseio de computadores e sistemas digitais.

Dentro do campo da Ciência da Computação, uma das áreas que tem como interesse tornar um sistema interativo mais eficiente e melhorar sua qualidade de uso é a Interação Humano-Computador (IHC). Hewett et al. (1992) definem a Interação Humano-Computador como uma disciplina que se ocupa do projeto, implementação e avaliação de sistemas computacionais interativos para uso humano, em conjunto com os fenômenos relacionados com esse uso. Os objetos de estudos de IHC podem ser inter-relacionados e agrupados em: a natureza da interação humano-computador; o uso de sistemas interativos situado em contexto; as características humanas; a arquitetura de sistemas computacionais e da interface com usuários; e os processos de desenvolvimento preocupados com o uso.

Para lidar com estas implicações, segundo Barbosa e Silva (2010), a IHC se apropria de conhecimentos e métodos de outras áreas para entender melhor os fenômenos envolvidos no uso dos sistemas computacionais. As áreas da Psicologia, Sociologia e Antropologia contribuem para aquisição de conhecimento sobre a cultura, o comportamento e os discursos dos usuários, e áreas como Design, Ergonomia, Linguística e Semiótica trazem conhecimentos e técnicas para melhorar a comunicação e interação do sistema com o usuário.

$\mathrm{Na}$ área de $\mathrm{IHC}$, apreender os conceitos de interface, interação e qualidade de uso é importante. A interface, conforme Preece et al. (1994), é a parte do sistema computacional com o qual o usuário se comunica, ou seja, aquela parte do sistema com a qual o usuário mantém contato físico para disparar as ações desejadas do sistema e receber os resultados dessas ações, que o usuário interpreta para em seguida definir suas próximas ações. A este processo de comunicação entre usuário e sistema se dá o nome de interação.

Ao projetar um sistema interativo, uma das preocupações do designer deve ser com a qualidade de uso relacionada à interação do usuário com o sistema.
Segundo Barbosa e Silva (2010), os critérios de qualidade de uso evidenciam certas características da interação e da interface que as tornam adequadas aos resultados esperados do uso do sistema. Alguns dos critérios de qualidade de uso são: usabilidade, experiência do usuário, acessibilidade e comunicabilidade. A usabilidade é um conceito que está relacionado com a facilidade de aprendizado e uso da interface. A qualidade denominada experiência do usuário engloba os sentimentos e as emoções dos usuários relacionadas ao uso dos sistemas computacionais interativos. O critério de acessibilidade está relacionado à remoção das barreiras que impedem mais usuários de serem capazes de acessar a interface do sistema e interagirem com ele. A comunicabilidade diz respeito à capacidade da interface de comunicar ao usuário as intenções do designer e os princípios de interação definidos durante o processo de design. No entanto, usabilidade é o critério de qualidade de uso mais conhecido e atualmente o mais utilizado tanto por pesquisadores quanto por profissionais da área, inclusive, esta qualidade de uso chega a ser sinônimo de interação.

\section{Integração das áreas de recuperação da informação, information search behaviour e interação humano-computador}

Os pesquisadores da Ciência da Informação estão procurando demonstrar a importância da sinergia entre as áreas de Rl, Comportamento de Busca de Informação e IHC, ou pelo menos entre duas destas áreas para criar sistemas altamente interativos, alcançar avanços substanciais nas capacidades de busca de informação e navegação, criar interfaces que suportam adequadamente a formulação de estratégias e a reformulação de buscas, tornarem mais fácil para os usuários recuperar a informação que precisam e auxiliar na compreensão do conjunto de informações localizadas, conforme Bohmerwald (2005); Ferreira e Pithan (2005); Marchionini (2006); Wildemurth (2006); Costa (2008); Keshavarz (2008); Mamoon et al. (2013); Joseph et al. (2013).

Ingwersen e Jarvelin (2005) analisaram os objetivos das investigações sobre Comportamento de Busca de Informação, Recuperação da Informação e Sistemas de Informação e observaram pouca sobreposição entre as três disciplinas, que seguiram suas próprias agendas 
de pesquisa. Ainda concluíram que as pesquisas sobre comportamento de busca de informação têm sido bem sucedidas até agora no desenvolvimento de uma compreensão teórica do processo de busca e em fornecer uma compreensão empírica dos fenômenos subjacentes, mas ficaram aquém no apoio a gestão da informação e no design de sistemas. A razão para isso está no foco da pesquisa, que geralmente exclui o sistema. Em contraste, quando o SRI está no centro da investigação e a interação é o foco da pesquisa, os usuários estão envolvidos, mas a amostra é geralmente oportunista (por exemplo, estudantes recrutados na instituição) e o contexto não é real. Esta descontextualização falha em capturar a relação da qualidade da informação com o seu uso ou o processo que desencadeou a atividade de pesquisa.

O campo da RI tem dado pouca atenção às questões de interface e, por outro lado, a área de IHC embora tenha amadurecido como disciplina ainda não realizou a integração com a pesquisa na área da Rl. Esperava-se que com as interfaces baseadas na Web haveria uma melhora na busca de informação para os usuários finais, entretanto os estudos realizados sobre a usabilidade desses sistemas foram suficientes para demonstrar que as dificuldades fundamentais de recuperação da informação ainda permanecem (Ahmed et al., 2009).

Ruthven (2008) afirma que embora a IHC e RI venham de diferentes tradições, por exemplo, enquanto $I H C$ dá mais ênfase à literatura publicada sobre usabilidade, a Rl enfatiza a eficácia. Ambas são importantes, pois um sistema com baixa usabilidade normalmente têm baixa eficácia e desenvolvedores de sistemas de baixa eficácia provavelmente pouco se importaram com a usabilidade deste sistema. Portanto, as áreas de IHC e RI podem aprender muito uma com a outra e o melhor da pesquisa em Rl freqüentemente refletirá em melhores práticas em IHC.

De acordo com Kelly e Sugimoto (2013), a combinação entre os campos da Recuperação de Informação, Comportamento de Busca de Informação e Interação Humano-Computador para formar uma especialidade única de pesquisa que visa capacitar as pessoas a explorar, resolver e gerenciar seus problemas de informações através das interações com os sistemas de informação é denominada de Recuperação de Informação Interativa (RII).

\section{Recuperação da informação interativa}

Esta área tem sido difundida através de várias instâncias práticas e acadêmicas. Os principais periódicos de RII são Journal of the American Society for Information Science and Technology, Information Processing \& Management, Journal of Documentation, Journal of Information Retrieval, e ACM Transactions on Information Systems, onde se publicam todos os artigos regularmente presentes na pesquisa de alta qualidade em RII. Da mesma forma o fazem os principais periódicos em Interação Humano-Computador, incluindo ACM Transactions on Computer-Human Interaction, Interacting with Computers e, em menor extensão, Human-Computer Interaction (Ruthven, 2008).

A Recuperação de Informação Interativa também conhecida como Recuperação da Informação Humano-Computador, conforme Marchionini (2006), preocupase com o estudo e a avaliação da interação dos usuários com sistemas de recuperação da informação e sua satisfação com a informação recuperada. Esta interação implica no envolvimento do usuário em contraste ao foco somente na recuperação da informação no qual aponta a abordagem de avaliação orientada ao sistema. A história da pesquisa na RI pode ser traçada por volta de 1953 e estabeleceu-se como uma disciplina empírica, mas a constituição da área de pesquisa de RIl é relativamente recente e tem o seu início nos anos 90 (Borlund, 2013).

De acordo com Belkin (2010), é bastante provável que o estado de conhecimento do usuário de informação se altere no decurso do episódio de busca de informação. A perspectiva de avaliação do paradigma tradicional de RI não leva em conta estes tipos de alterações, pois não responde a natureza da RII, em termos dos tipos de metas que a busca de informação pressupõe, em termos de sua incapacidade de avaliar os episódios inteiros de busca de informação e em termos da sua incapacidade para levar em conta as alterações do pesquisador que são inerentes à busca de informação interativa.

As demandas por abordagens que considerem os aspectos humanos em recuperação da informação têm sido difundidas devido a predominantemente a três mudanças realizadas na literatura ao buscarem novas interpretações para caracterizar as necessidades informacionais, apontadas por Robertson e Hancock-Beaulieu 
(1992): (a) perspectiva da relevância, ou seja, a relevância de um objeto informacional deve ser medida em relação ao potencial para atender uma necessidade informacional; (b) perspectiva cognitiva, no qual compreende que uma lacuna no conhecimento do usuário pode levá-lo a interagir com um sistema de RI e de acordo com a gravidade desta lacuna, ele pode não conseguir elaborar satisfatoriamente uma consulta no sistema; e a (c) perspectiva interativa, em que deve ser considerado não apenas a relação consulta-documentos, mas também as interações do usuário com o sistema e os documentos, além do comportamento das reformulações da consulta.

Segundo Kelly (2009), uma maneira de pensar sobre a RIl é colocá-la no meio de um continuum que de um lado examina os estudos sob a ótica do sistema e do outro analisa os estudos focados nos usuários. Os estudos situados na direção dos sistemas:

Estudos TREC 3 : estão focados no desenvolvimento e avaliação de algoritmos de recuperação e técnicas de indexação. Nestes estudos, não há a participação de usuários reais;

Avaliação da relevância: empregam os usuários para avaliar a relevância dos documentos em relação às suas tarefas. No entanto, nestes estudos os usuários estão presentes apenas para auxiliar na construção de infra-estrutura, não há um interesse pelas experiências e comportamentos de busca dos usuários e suas interações com os sistemas;

Avaliação de sistemas de disseminação seletiva de informações: oriundos da década de 70, o modelo de avaliação destes estudos era selecionar usuários com perfis semelhantes para avaliar os documentos que foram apresentados para os usuários através da determinação do perfil;

Análises de Logs: usam logs de transações para identificar regularidades e monitorar alguns aspectos da experiência de busca do usuário com o sistema;

Em seguida, Kelly (2009) apresenta os estudos situados na direção dos usuários:

Estudos da TREC Interativa: uma característica do sistema ou interface é tipicamente avaliado e o design deste sistema geralmente está baseado nas atividades de cognição e nos comportamentos humanos envolvidos na busca de informação. Os estudos localizados em torno deste ponto empregam vários métodos de coleta de dados, e normalmente incluem medidas de desempenho do sistema, interação e usabilidade. Este ponto representa o estudo clássico ou arquetípico da RII;

Estudos sobre comportamento informacional: o ponto principal de tais estudos é isolar e estudar os aspectos individuais dos usuários no processo de pesquisa e fazer com que essas experiências sejam tão semelhantes quanto possível, de modo que a causalidade pode ser estudada com maior confiança. Estes estudos são geralmente mais interessados em dizer alguma coisa específica sobre o comportamento, em vez de demonstrar a qualidade de uma característica ou sistema de RII;

Estudos de comportamento de busca com SRI: estas avaliações podem incluir estudos das táticas de busca dos usuários, estudos de como os usuários fazem avaliações de relevância ou como os usuários encontram as informações na Web, também podem incluir pesquisadores intermediários e outros profissionais. Estes estudos muitas vezes procuram entender o comportamento natural de busca dos usuários, que é fundamental para o desenvolvimento de melhores sistemas de RII. No entanto, esses estudos nem sempre são impulsionados por preocupações com o sistema;

Estudos de comportamento de busca de informação: podem ser caracterizados por avaliações que se concentram quase exclusivamente nos seres humanos, suas necessidades e comportamentos informacionais. Nestes estudos, os investigadores exploram as necessidades de informação de usuários reais e sua posterior busca da informação, no contexto particular em que essas necessidades surgem sem relação a um tipo particular de sistema de RI.

A área de RIl estabeleceu um novo rumo dentro da longa tradição de RI, ao longo das duas últimas décadas, que introduz o usuário em seu centro e coloca novos desafios para avaliação do sistema. O desempenho dos SRI pode ser melhorado através do uso de informações sobre todo o processo interativo de pesquisa. No entanto,

\footnotetext{
3 A Text Retrieval Conference (TREC), co-patrocinado pelo National Institute of Standards and Technology (NIST) e U.S. Department of Defense, foi iniciada em 1992 como parte do TIPSTER Text Program. Seu objetivo é apoiar a investigação junto com a comunidade de Recuperação de Informação, fornecendo a infraestrutura necessária para a avaliação em larga escala de metodologias para recuperação de texto.
} 
esta abordagem até agora foi só inicialmente explorada e possui grande potencial de pesquisa para o futuro (Bierig et al., 2010).

O potencial de uma boa pesquisa nesta área não vem só a partir do conhecimento técnico no desenvolvimento de sistemas interativos, mas também a partir do conhecimento sobre o comportamento de busca de informação das pessoas e enquanto interagem com os sistemas de busca de informação. Atualmente, os pesquisadores que se interessam pelos estudos dos sistemas de busca têm privilegiado a perspectiva do usuário, concentrando em suas necessidades e comportamentos de informação para projetar interfaces de busca que suportem ricas interações.

\section{Conclusão}

Apesar da realização de vários estudos através de diversas abordagens já terem sido adotadas para avaliar e propor melhorias para o aperfeiçoamento dos SRI, ainda é um desafio para a pesquisa em RI desenvolver pesquisas e soluções para o desenvolvimento de sistemas que sejam capazes de recuperar informações relevantes de modo mais fácil para os usuários.

Os usuários ainda esperam que vários destes sistemas Ihes permitam concentrar-se apenas na busca e não exijam deles a preocupação em aprender a usá-los. Assim, através do aumento da qualidade de uso dos

\section{Referências}

Ahmed, S.M.Z.; Mcknigh, C.; Oppenheim, C. A review of research on human-computer interfaces for online information retrieval systems. The Electronic Library, v.27, n.1, p.96-116, 2009. Available from: <http://www.emeraldinsight.com/ journals.htm?articleid=1771204>. Cited: Sept. 15, 2013.

Barbosa, S.D.F.; Silva, B.S. Interação humano-computador. Rio de Janeiro: Elsevier, 2010.

Belkin, N.J. On the evaluation of interactive information retrieval systems. In: Larsen, B. (Ed.). The Janus faced scholar: A festschrift in honor of Peter Ingwersen. Conpenhagen: Det Informations Videnskabelige Akademi, 2010. p.13-21. Available from: <http://vbn.aau.dk/files/90357690/JanusFacedScholer Festschrift_Peterlngwersen_2010.pdf\#page $=16$ >. Cited: Jan. 13, 2014.

Bierig, R.; Cole, M.; Belkin, N. A data analysis and modelling framework for the evaluation of interactive information retrieval. In: Gurrin, C. (Ed.). European Conference on IR
SRI é possível proporcionar maior apoio aos usuários diminuindo os erros cometidos, reduzir os custos com treinamentos e suporte técnico, tornar mais agradável à experiência de uso e aumentar o sucesso dos SRI para serem realmente úteis.

Na área da Ciência da Informação, pesquisadores tentam demonstrar a importância da coesão entre as áreas de RI, Comportamento de Busca de Informação e IHC para alcançar avanços substanciais nas capacidades de busca de informação, formulação de estratégias e navegação dos SRI, pois o processo de busca de informação é complexo, multidimensional e possível de ser investigado a partir de várias perspectivas.

O campo de pesquisa RII pondera sobre a necessidade de alcançar novas interpretações das demandas informacionais e avaliar o episódio da busca de informação de modo mais abrangente levando em conta as várias alterações realizadas pelos usuários. Esta abordagem traz novos desafios para avaliação dos SRI, foi pouco explorada e ainda possui enorme potencial de pesquisa.

Além disso, é importante que as organizações e os projetistas responsáveis por desenvolver os SRIs estejam conscientes da necessidade de trabalharem em parceria com os pesquisadores para realizarem intervenções adequadas e aperfeiçoarem estes sistemas, e por intermédio de investimentos em suportes e novos recursos para busca de informação possam resolver realmente os problemas dos usuários e ajudá-los a alcançarem seus propósitos.
Research, 32., 2010, Berlim. Advances Information Retrieval. Berlin: Springer-Verlag, 2010. p.673-674. Available from: <http:// comminfo.rutgers.edu/ jacekg/pubs/txt/2010_ECIR_ Analysis_system_RBierig_etal.pdf >. Cited: Dec. 23, 2013.

Bohmerwald, P. Uma proposta metodológica para avaliação de bibliotecas digitais: usabilidade e comportamento de busca por informação na biblioteca digital da PUC-Minas. Ciência da Informação, v.34, n.1, p.95-103, 2005.

Borlund, P. Interactive information retrieval: An introduction. Journal of Information Science Theory and Practice, v.1, n.3, p.12-32, 2013. Available from: <http://central.oak.go.kr/ repository/journal/12443/E1JSCH_2013_v1n3_12.pdf>. Cited: Jan. 5, 2014.

Branski, R.M. Recuperações de informações na web. Perspectivas em Ciência da Informação, v.9, n.1, p.70-87, 2004. Disponível em: <http://portaldeperiodicos.eci.ufmg.br/index.php/pci/ article/view File/351/160>. Acesso em: 2 dez. 2013. 
Costa, L.F. A usabilidade do Portal de periódicos da CAPES. 2008. Dissertação (Mestrado em Ciência da Informação) - Universidade Federal da Paraíba, 2008. Disponível em: <http:// dci2.ccsa.ufpb.br:8080/jspui/bitstream/123456789/99/3/ Disserta\%C3\%A7\%C3\%A30\%20Luciana\%20Costa.pdf>. Acesso em: 20 jun. 2012.

Ferreira, S.M.; Pithan, D.N. Estudo de usuários e usabilidade na biblioteca INFOHAB: relato de experiência. In: Simpósio Internacional de Bibliotecas Digitais. 3., São Paulo, 2005. Anais eletrônicos... Disponível em: <http://eprints.rclis.org/handle/ 10760/10483? mode=full\#.T9aMcWGTIJE $>$. Acesso em: 11 maio 2012.

Hewett, T.T. et al. ACM SIGCHI curricula for human-computer interaction. New York: ACM, 1992. Available from: <http://old. sigchi.org/cdg/>. Cited: Sept. 11, 2013.

Ingwersen, P., Jarvelin, K. The turn: Integration of information seeking and retrieval in context. Dordrecht: Springer, 2005.

Joseph, P. et al. Models of information search: A comparative analysis. Information Research, v.18, n.3, 2013. Available from: <http://informationr.net/ir/18-1/paper562.html\#.uoggy ha3tc1>. Cited: Nov. 13, 2013

Kelly, D. Methods for evaluating interactive information retrieval systems with users. Foundations and Trends in Information Retrieval, v.3, n.1-2, p.1-224, 2009. Available from: <http://ils.unc.edu/ dianek/FnTIR-Press-Kelly.pdf>. Cited: Dec. 22, 2013.

Kelly, D.; Sugimoto, C.R. A systematic review of interactive information retrieval evaluation studies, 1967-2006. Journal of American Society for Information Science and Technology, v.64, n.4, p.745-770, 2013.

Keshavarz, H. Human information behaviour and design, development and evaluation of information retrieval systems. Program: Electronic Library and Informations systems, v.42, n.4, p.391-401, 2008. Available from: <http://www.emeraldinsi ght.com/journals.htm?articleid=1748055\&show=abstract $>$. Cited: Dec. 20, 2013.

Mamoon, M.H.; El-Bakri, H.M.; Salama, A.A. Interactive visualization of retrieved information. International Journal of Knowledge Engineering and Research, v.2, n.4, p.217-231, 2013.
Available from: <http://www.ijker.org/vol2issue4/paper 24.pdf>. Cited: Dec. 10, 2013.

Marchionini, G. Towards human-computer information retrieval. Bulletin of the American Society for Information Science and Technology, n.32, p.20-22, 2006. Available from: <http:// www.asis.org/Bulletin/Jun-06/marchionini.html>. Cited: Sept. 19, 2013.

Preece, J. et al. Human-computer interaction. Wokingham: Addison-Wesley, 1994.

Robertson, S.E.; Hancock-Bealieu, M.M. On the evaluation of IR systems. Information Processing \& Management, v.28, n.4, p.457-466, 1992.

Ruthven, I. Interactive information retrieval. Annual Review of Information Science and Technology, v.42, n.1, p.43-91, 2008. Avaialble from: <http://onlinelibrary.wiley.com/doi/10. 1002/ aris.2008.1440420109/pdf>. Cited: Dec. 20, 2013.

Souza, R.R. Sistemas de recuperação das informações e mecanismos de busca web: panorama atual e tendências. Perspectivas em Ciência da Informação, v.11, n.2, p.161-173, 2006. Disponível em: <http://www.scielo.br/scielo.php? script=sci_arttext\&pid=S1413-99362006000200002 $>$. Acesso em: 2 dez. 2013.

Torres Pombert, A. El uso de los buscadores en Internet. Revista de Ciencias Médicas de La Habana, v.11, n.3, 2003. Disponible en: <http://bvs.sld.cu/revistas/aci/vol11_3_03/aci04303. htm>. Acceso en: 8 dec. 2013.

Wildemurth, R. Evidence-based practice in search interface design. Journal of American Society for Information Science and Technology, v.57, n.6, p.825-828, 2006. Available from: <http://onlinelibrary.wiley.com/doi/10.1002/asi.20301/pdf>. Cited: Sept. 3, 2013

Wilson, T.D. Models in information behavior research. The Journal ofDocumentation, v.55, n.3, p.249-270, 1999. Available from: <http://www.informationr.net/tdw/publ/papers/1999 JDoc.html>. Acesso em: Sept. 5, 2013.

Xie, I. Information searching and search models. In: Bates, J.M.; Maak, M.N. (Ed.). Encyclopedia of Library and Information Sciences. $3^{\text {rd }}$ ed. New York: CRC Press, 2010. p.1-13. Available from: <https://pantherfile.uwm.edu/hiris/www//risArticles/ Articles/120043745.pdf>. Cited: Dec. 11, 2013. 
